\title{
The greenBag, the New Solution in Waste Separation
}

\author{
Paolo Sperandio ${ }^{\#}$, Massimiliano De Luca ${ }^{*}$, Alexandro Catini ${ }^{+}$ \\ \# aTon S.r.L., Via Adriano 5, Guidonia (Rome), 00012, Italy \\ E-mail: p.sperandio@aton-srl.it

\begin{abstract}
*CNR INM Institute of Marine Engineering, Research Unit Acoustics, and Sensors "O.M. Corbino" Research Area Tor Vergata, Via Fosso del Cavaliere 100, Rome, 00133, Italy

E-mail: massimiliano.deluca@cnr.it
\end{abstract}

${ }^{+}$Department of Electronic Engineering of University of Rome Tor Vergata, Via del Politecnico 1, Rome, 00133, Italy E-mail: catini@ing.uniroma2.it

\begin{abstract}
The greenBag is an innovative households' Used Cooking Oil (UCO) disposal solution aiming to allow the collection of this feedstock interesting European but also Chinese and US market. The possibility to collect UCO produced by households represents the possibilities to increase the amount of biodiesel, respecting the ILUC directive, of the $60 \%$. So far, there are no concrete solutions able to properly respond to this need, due to their huge prizes and due to the low performances, they provide. greenBag is a solution coming from an intensive research activity completed by an infield validation. It has been designed to reply to all the requirements coming from families and from the waste management companies, the two actors involved in UCO disposal. Indeed, two different types of users in general, and in particular the UCO collection, characterize waste collection solutions: families - that want simple solutions, easy to use and able to reward them; waste collection companies - that want cheap solutions, able to trace waste disposals and able to assure them a high quality of wastes. The currently available market solutions are all focused on waste companies and the current innovative ideas designed by Universities and Startups focus their attention only on one of the two involved actors. greenBag is currently the sole solution that aims to satisfy both actors, exploiting the disposal traceability not only to know how many waste people produces but also to quantify their efforts and making them aware about the overall disposal process.
\end{abstract}

Keywords - UCO; used cooking oils; biodiesel; circular economy; separation of waste.

\section{INTRODUCTION}

An important problem for our society is the waste management for rubbish produced in the world. An effective solution for several goods such as paper, plastic, PVC and other homogenous products is represented by the recycle [14]. Among these, the Used Cooking Oil (UCO) recycle is gaining great attention. Currently, the households' Used Cooking Oil (UCO) collection market is characterized by huge costs represented by Industrial costs, Logistic costs, and Social costs.

The contamination of UCO with other liquids that could be mineral oils, paints, acids, etc. oblige UCO collection companies to filter this liquid before selling it or, in the worst cases to dispose of it after having collected it. This UCO contamination currently is unavoidable; the most used collection technologies and methodologies. Door to door, collection and proximity collection do not foresee any check of the liquid, and any tracking of the citizens' provisions produce huge costs. It is not only to collect and to transport these liquids in their headquarters but also implementing expensive techniques to filter them or in the worst cases to properly dispose of them.

Households' UCO collection is based on periodic checks of the containers deployed inside the Municipalities. Indeed employees of the UCO collection company periodically. Collect plastic containers from families giving them back new clean ones (door-to-door collection). Check the outdoor containers to verify the availability of UCO inside them (proximity collection). Both methodologies introduce fixed costs like employees, fuel, cleaning costs of the provided containers that affect the revenues of UCO collection companies.

UCO nowadays is generally disposed of households using sewer pipes. The majority of people are not informed about where UCO have to be disposed and how to dispose of it, so they prefer to trash it using the drainage system, also to avoid taxes to pay in case of errors during the separation of waste. Such behavior unable to be controlled and produces enormous problems. Citizens, creating obstructions in 
sewage pipes complicated to remove; the exception has done with costly and specific interventions aiming to clean the pipes and/or to replace them. Environment, UCO creates thin films over the water that act like a dark shield that reduces the amount of light necessary for marine life. The UCO that instead is through on the soil creates a waterproof layer that imped the exchange of nutrients with the plants

These different costs represent the main issues affecting the households' UCO collection market, a market that currently is not supported by solutions able to reply to the different needs coming out from citizens and UCO collectors. The unavailability of a reliable and economical solution for the households' UCO collection is affecting a market with tremendous opportunities, in TABLE 1 we reported the amount of UCO produced and collected in each EU country, according to [5].

TABLE 1

UCO PRODUCTION AND COLLECTION IN EU

\begin{tabular}{|l|r|r|c|}
\hline \multicolumn{1}{|c|}{ Country } & $\begin{array}{c}\text { UCO } \\
\text { collectible } \\
\text { household } \\
\text { resources }\end{array}$ & Collected & $\begin{array}{c}\text { UCO \% } \\
\text { collected }\end{array}$ \\
\hline Italy & 156,000 & 15,000 & $9.6 \%$ \\
\hline Germany & 65,000 & 1,209 & $1.9 \%$ \\
\hline France & 52,000 & 0 & $0.0 \%$ \\
\hline Spain & 232,000 & 5,000 & $2.2 \%$ \\
\hline Romania & 49,000 & 0 & $0.0 \%$ \\
\hline Poland & 47,000 & 0 & $0.0 \%$ \\
\hline UK & 42,000 & 8,600 & $20.5 \%$ \\
\hline Hungary & 29,000 & 40 & $1.4 \%$ \\
\hline Bulgaria & 27,000 & 0 & $0.0 \%$ \\
\hline Portugal & 30,000 & 1,000 & $3.3 \%$ \\
\hline Czech Republic & 16,000 & 500 & $3.1 \%$ \\
\hline Croatia & 12,000 & 0 & $0.0 \%$ \\
\hline Belgium & 13,000 & 8,300 & $63.8 \%$ \\
\hline Slovakia & 10,000 & 360 & $3.6 \%$ \\
\hline Netherlands & 12,000 & 3,600 & $30.0 \%$ \\
\hline Austria & 7,000 & 2,352 & $33.6 \%$ \\
\hline Greece & 20,000 & 14 & $0.1 \%$ \\
\hline Lithuania & 6,000 & 0 & $0.0 \%$ \\
\hline Latvia & 4,000 & 0 & $0.0 \%$ \\
\hline Estonia & 4,000 & 0 & $0.0 \%$ \\
\hline Slovenia & 4,000 & 0 & $0.0 \%$ \\
\hline Finland & 3,000 & 0 & $0.0 \%$ \\
\hline Sweden & 3,000 & 1,400 & $46.7 \%$ \\
\hline Denmark & 2,000 & 1 & $0.1 \%$ \\
\hline Ireland & 2,000 & 0 & $0.0 \%$ \\
\hline Cyprus-modelled & 4,0000 & 0 & $0.0 \%$ \\
\hline Malta-modelled & 2,000 & $0.0 \%$ \\
\hline Luxemburg-modelled & 1,000 & $0.0 \%$ \\
\hline Total & $\mathbf{8 5 4 , 0 0 0}$ & $\mathbf{4 7 . 7 3 6}$ & $\mathbf{5 . 6 \%}$ \\
\hline & & & \\
\hline
\end{tabular}

Considering that the auctions of UCO can span from $€$ 600,00 per ton up to $€ 1.000,00$ per ton $\left(1 \mathrm{~m}^{3}=1\right.$ ton $)$, the so far neglected market is moving from $€ 483.758 .400,00$ up to $€$ 806.264.000,00 per year (only in EU). UCO is an important feedstock recognized by European Commission thanks to RED (Renewable Energy Directive) and FQD (Fuel Quality Directive). They are EU directives respectively asserting that for 2020 in each Member State the $10 \%$ of the energy consumed by road transports has to come from renewable sources and that during their life cycle, fuels have to produce $10 \%$ less of $\mathrm{CO} 2$ in comparison with 2010 and that at least $60 \%$ of this reduction should be obtained using bio-fuels. But also among the biofuels that can be produced in respect with RED and FQD the EU recognizes a special importance to UCO with ILUC (Indirect Land Use Change), establishing that biofuel from Used Cooking Oils (UCO), animal fats, non-food cellulosic material, ligno-cellulosic material except saw logs and veneer logs will count twice towards the targets. Indeed an issue being debated in the past years is the risk of deliberate "production" of UCO. There is a risk of fraud when the economic value of UCO is higher than virgin oils due to the above-mentioned double counting measure that provide extra incentives to use UCO for biofuel production. Since it could be very difficult to trace the origins of the UCO, there is a risk of deliberate production of waste and imports of poorly checked waste [6].

In Germany, the UCO used as biofuel feedstock must be traceable, starting from the origin up to and including the production of the biofuel, and requires ISCC DE certification. In the Netherlands, only the verification (not certification) of double counting following Dutch legislation is required and done at the biofuels producer. However, this certification is partially done for the UCO coming from Ho.Re.Ca. (Hotel, Restaurant and Catering) and food industries, it is not implemented for the UCO coming from households due to the tracking and issue.

To improve the quality of the collected waste, many solutions have been tested, some based on citizen's intentions, others based on the establishment of advanced technological solutions that include machine learning solutions [7]-[9], artificial intelligence based on dedicated how accelerators [10]-[12] and advanced telecommunications infrastructure [13], [14]. In this paper, we present the greenBag solution that is based on a telecommunication infrastructure and specially designed sensors.

\section{MATERIALS AND METHOD}

The greenBag is an innovative eco-solution designed for the collection of UCO (Used Cooking Oils) produced by households. It is based on innovations both in terms of technology (an HW/SW infrastructure able to make easy this type of collection) and of methodology (based on a pervasive installation of the containers and a strategy. It aims to improve people's education and participation, recognizing their duties but also their honors). greenBag solution is formed by three main elements.

\section{A. The greenBag Bin}

The greenBag Bin comprises a metal shell including three different inner tanks:

1) Analysis box: a 5 liters tank closed by an electric lock that could be opened only using a pre-registered NFC card. The analysis box is equipped with a smell reduction system, formed by active carbons filters able to reduce UCO smell and with different sensors able to analyses the liquids provided by households. At the bottom of the analysis box, there are two different electro valves, controlled by a microcontroller, that connect the analysis box with other two 
different tanks: the UCO Tank and the Scrap Tank. Depending on the result obtained from the sensors the microcontroller will release one of the two electro valves in order to keep the UCO separated from the Scrap

2) UCO Tank: a metal tank of 400x300x800 mm, ca. 100 liters, equipped with a set of four wheels, allowing the movement of the tank; an ultrasound sensor, able to evaluate the level of the liquid in the tank. The tank is completed with a brake system, which increase its stability, and with handles, that simplify its movement

3) Scrap Tank: a metal tank of 300x150x800 mm, ca. 40 liters, equipped with a set of four wheels, allowing the movement of the tank; an ultrasound sensor, able to evaluate the level of the liquid in the tank. The tank is completed with a brake system and a dynamic ballast necessary to assure its stability, due to the shape of the tank, Fig. 1 greenBag first prototype and Fig. 2 greenBag final prototype

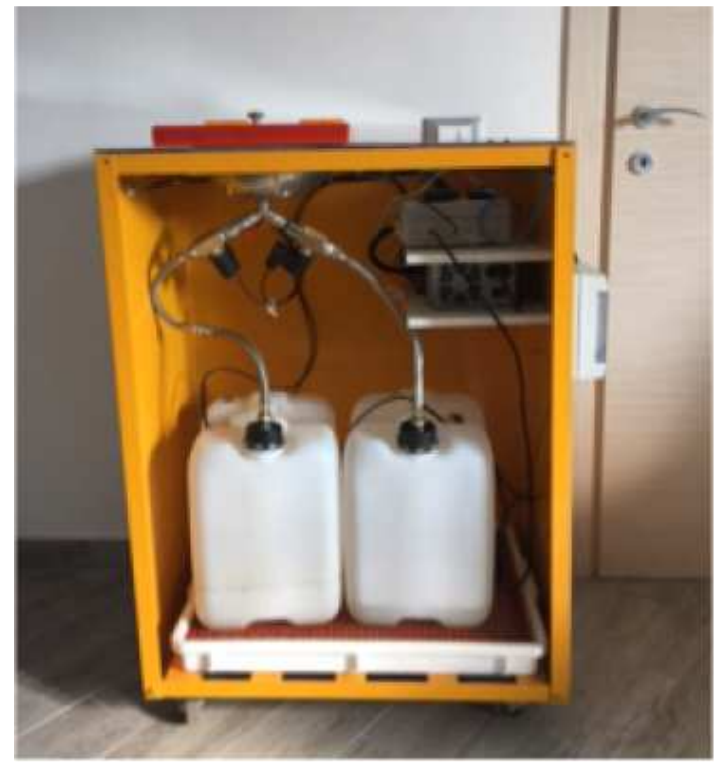

Fig. 1: greenBag first prototype

The greenBag bin is based on an embedded architecture similar to [15-17], since it allows to enter in deep sleep states and to save the device battery.

\section{B. The greenBag C2R (Command and Control Room)}

The greenBag C2R (Command and Control Room) is the back end module installed on a Cloud platform, it is connected with all the greenBag Bins deployed in the urban area. It receives, from each greenBag Bin, data about who provides liquids in the Bin, what amount, what quality, the level of the UCO Tank, the level of the Scrap Tank, a check about the status of all the hardware installed in each greenBag Bin. Collecting all those data, and analyzing them, the greenBag $\mathrm{C} 2 \mathrm{R}$ is able to provide an optimization of the transport route, assuring to UCO collectors always a full load for each vehicle used for the UCO collection and a consequent reduction of costs during transport phase. It also provides a complete analysis of what are the areas of the city providing the most amount of UCO but also which are the less efficient areas and where arrange initiatives to improve the citizens' participation

\section{The greenBag App}

The greenBag App is downloadable from the App markets. It is the front end of the greenBag platform, it allows its owner to know where greenBag Bins are, how much UCO he provided, his last UCO provisions, the initiative implemented by UCO collectors and the overall status of the UCO collection of his Municipality. Moreover, the App provides the opportunity to send alert messages (composed by images and texts) to the UCO collectors

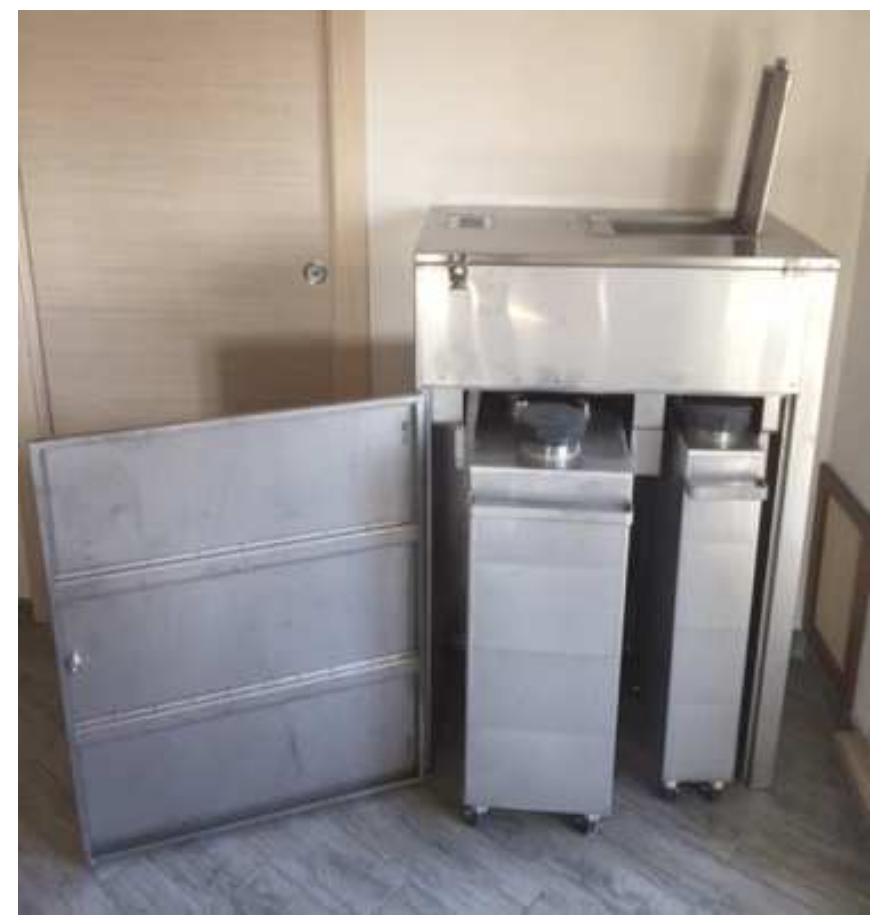

Fig. 2: greenBag second prototype

\section{RESULTS AND DISCUSSION}

The three-greenBag modules: the greenBag Bin, the greenBag $\mathrm{C} 2 \mathrm{R}$ and greenBag App are three different entities working togheter to provide not only UCO recognition but also UCO separation and waste disposal tracking.

Each module in greenBag has been deeply studied and implemented in order to solve a specific role, here after we provide a deep explanation of the technologies used and the service provided by each module.

\section{A. greenBag Bin}

The greenBag Bin is the core of the entire greenBag platform. As indicated in the previous section it is formed by different elements: three inner tanks able to gather the liquids provided by people, a motherboard able to collect the data during each UCO disposal, the sensors able to collect the data from UCO and send them over internet. The sensors represent the elements able to detect a physical quantity and transform it in electric signals that can be elaborated by a microcontroller. Since the beginning, to estimate the quality of the UCO, we worked with capacitive sensors. A capacitive sensor is a device whose physical characteristics 
determine the value of its capacitance. The capacitance value is altered when there is:

- An alteration of the separation distance between the plates

- A sliding of one of the plates, altering the common area between its surfaces

- Alterations of the dielectric element er between the plates

As well indicated by equation (1), reporting the reaction among capacitance and geometric parameters of a capacitor:

$$
C=\varepsilon_{0} * \varepsilon_{r} * \frac{S}{d}
$$

We are able to characterize the dielectric inside the plates of a capacitor. Indeed the capacitance relates to the electrical energy storage capacity between the plates of a capacitor when they are submitted to a difference in potential.

The capacitance $(\mathrm{C})$ between the two parallel plates of a capacitor, where $\varepsilon_{\mathrm{r}}$ is a relative dielectric constant that depends on the dielectric material, $\varepsilon_{0}$ is the constant of dielectric permissiveness in the vacuum $(8.8 \times 10-12)$ " $\mathrm{S}$ " is the plate's common area, and " $\mathrm{d}$ " is the separation distance between the plates.

Keeping constant:

- The areas of the capacitors' plates and

- The distances between plates

We are able to estimate the $\varepsilon_{\mathrm{r}}$.

Using this solution, not only with one capacitor but putting in parallel several of them, we are able to reliable identify the nature of the UCO. However, this solution, even if cheaper, presents several issues that cannot allow us to economically deploy it to the market:

- A huge calibration of the sensor is necessary

- The sensor is submerged inside the UCO so it has to be continuously cleaned

In order to avoid continuous cleaning of the sensor, we decided to move on an optical solution. In particular, using a micro spectrometer system, C12666 from Hamamatsu Fig. 3, and an electronic interface able to integrate an UV LED source, we are able to estimate the variation of a liquid fluorescence intensity over the entire spectrum. Exposing the used cooking oils to an UV radiation (peaked @ $400 \mathrm{~nm}$ ) we obtained a fluorescence response with a maximum at $625 \mathrm{~nm}$ typical for the olive oil, see Fig. 4, developed using a hardware architecture [18].

As indicated above the collection of UCO produced by households is critical because they use the provided containers to dispose also mineral oils that are not recyclable and are very difficult to separate by vegetable oils. Using our solution we are able to distinguish also mineral oils because the absorption response of the mineral oil is completely different from the one produced by vegetable oils, see Fig. 7. Therefore using this optical solution, we are able to distinguish among different type of oils.

We would like to underline that the aim of the greenBag platform is to identify a liquid disposed inside from people is UCO or not. It is not a platform developed to certify if the oil is still edible or not. greenBag's role is to distinguish among oils and other liquids in an automatic and reliable way allowing the recycling of it in biofuel, glycerin and lubricants.

The results produced by the sensors, but also the information about who produced the UCO disposed and the current status of the greenBag Bin are all data managed by a microcontroller that can be sent over internet.

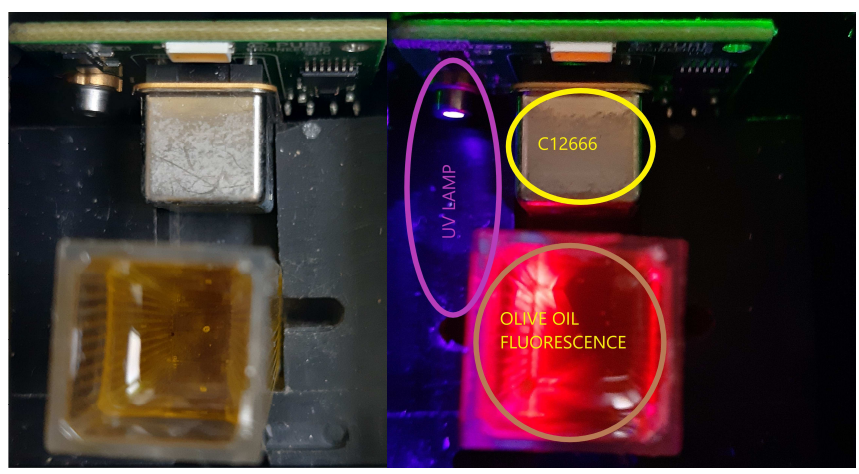

Fig. 3: Hamamatsu C12666 spectrometer fully integrated with UV laser diode. Oil fluorescence is shown.

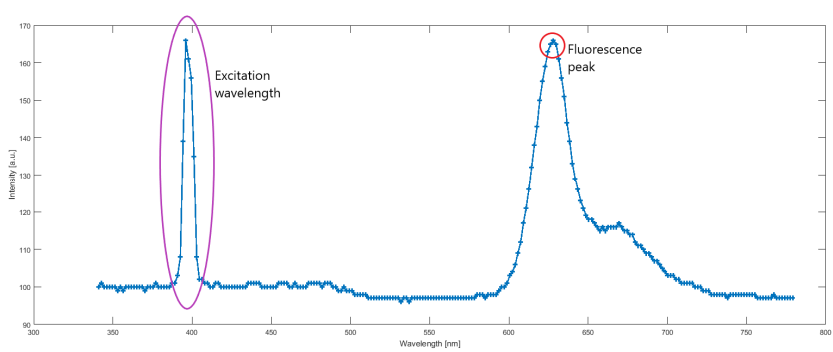

Fig. 4: Olive oil visible spectrum fluorescence

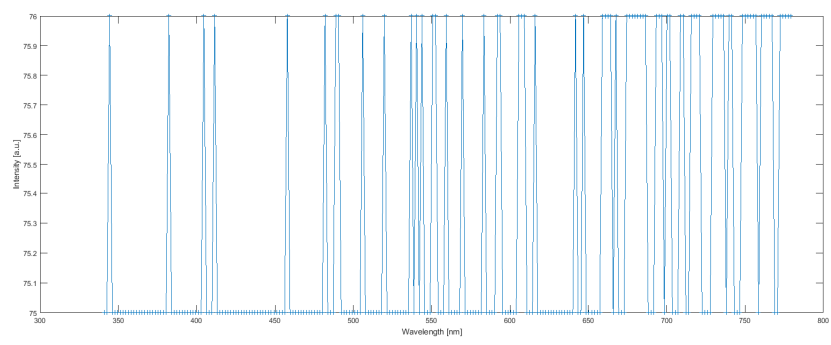

Fig. 5: Mineral oil visible spectrum (totally black)

The first greenBag prototype has been equipped with $3 \mathrm{G}$ router to be always connected. In analyzing the amount of data, it was easy to understand that a less bandwidth and the low energy consumption on radio technology can be adopted.

\section{B. greenBag $C 2 R$}

The C2R (Command \& Control Room) is a Cloud platform able to collect all the data coming from the greenBag Bins and to elaborate them similarly to [19-20].

The data coming out from each greenBag Bin are related to:

- The person who dispose liquids (to respect the privacy terms he is identified by a unique code)

- The type of liquid disposed, e.g. if it is UCO or not. As indicated in the previous section the sensors are able to recognise UCO, other type of liquids that do 
not match with UCO patterns are discarded and registered as Scrap

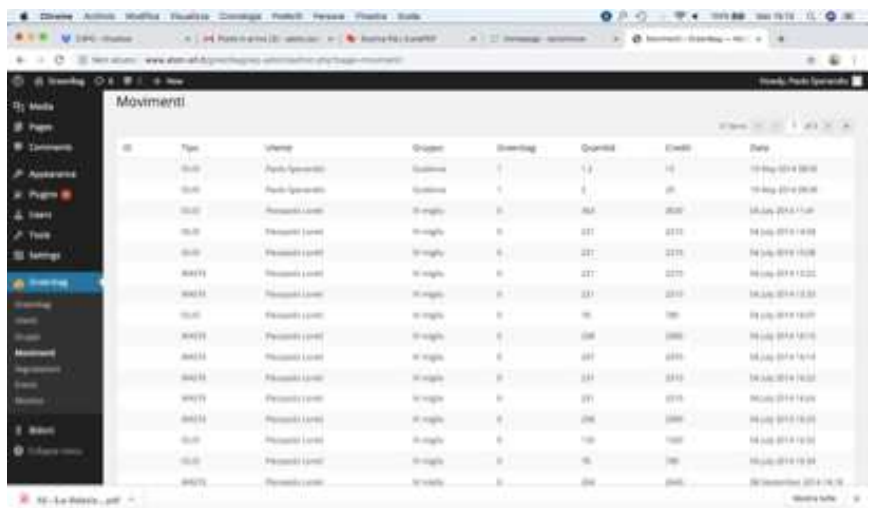

Fig. 5: C2R dashboard

- The amount of liquid disposed

- The data concerning the status of the greenBag Bin e.g. its position and the level of liquids inside both UCO and Scrap Tank. This information is particularly useful in order to identify proper routes able to improve the collection rate of the waste management company, that will start the UCO collection knowing a priori the amount of UCO contained in each Bin

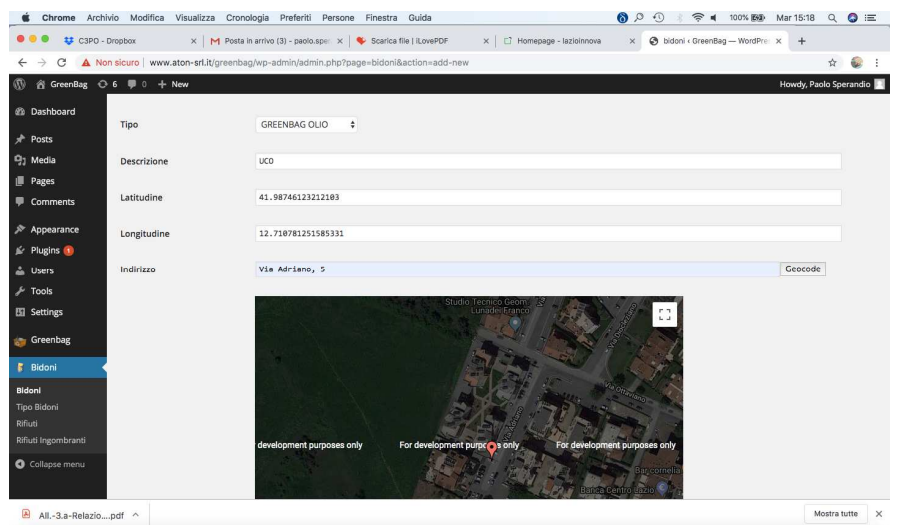

Fig. 6: $\mathrm{C} 2 \mathrm{R}$ greenBagBin position

All these data are used to create statistics about:

- The rate of waste disposal of each citizen

- The evaluation of the waste tax. One of the main objective in this sense is to provide a valid tool able to exactly know the amount of waste produced

- Understand people behaviour and try to improve their effort arranging spefic events, workshops, etc.

\section{C. greenBag App}

The last greenBag module is represented by its App. It is the front end with citizens, and it allows them to control:

- Their disposal rate

- Where greenBag Bins are

- A waste dictionary

- Other set of functionalities able to improve their efforts like news about the waste collection methodology, monthly report about their disposal, municipality waste separation rate, etc.

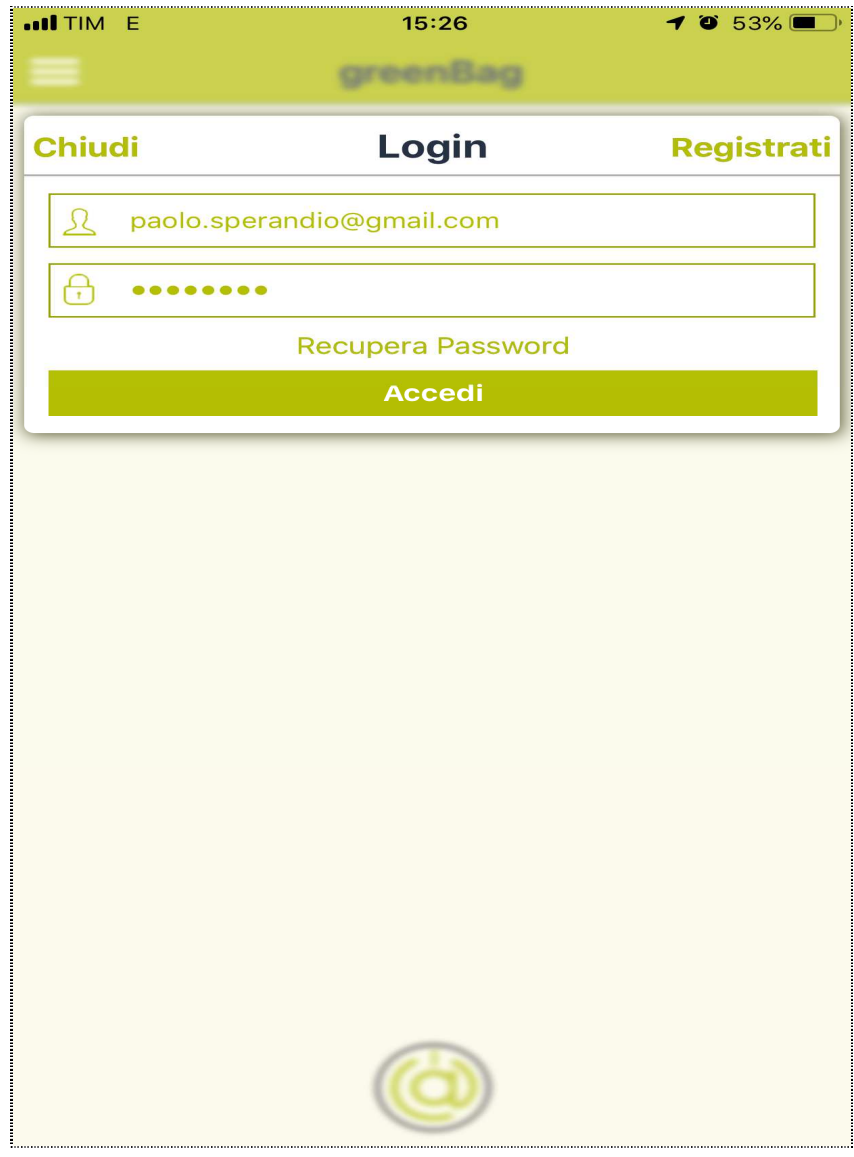

Fig. 7: greenBag App Login
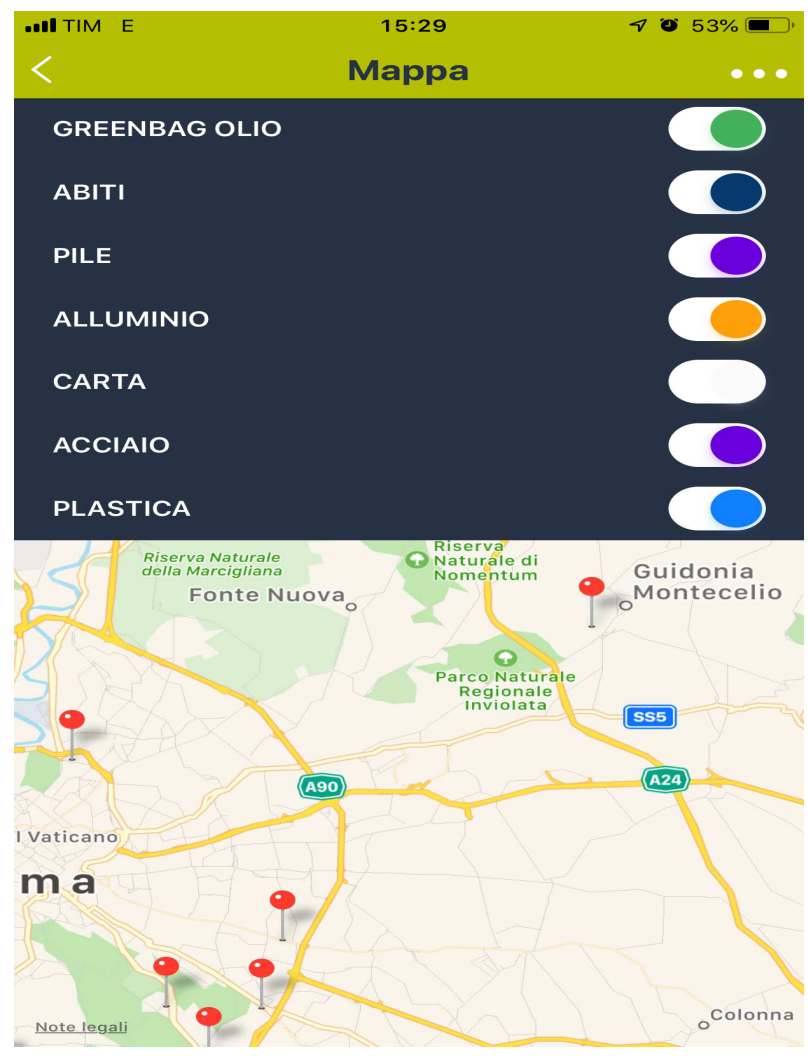

Fig. 8: greenBag App Map 


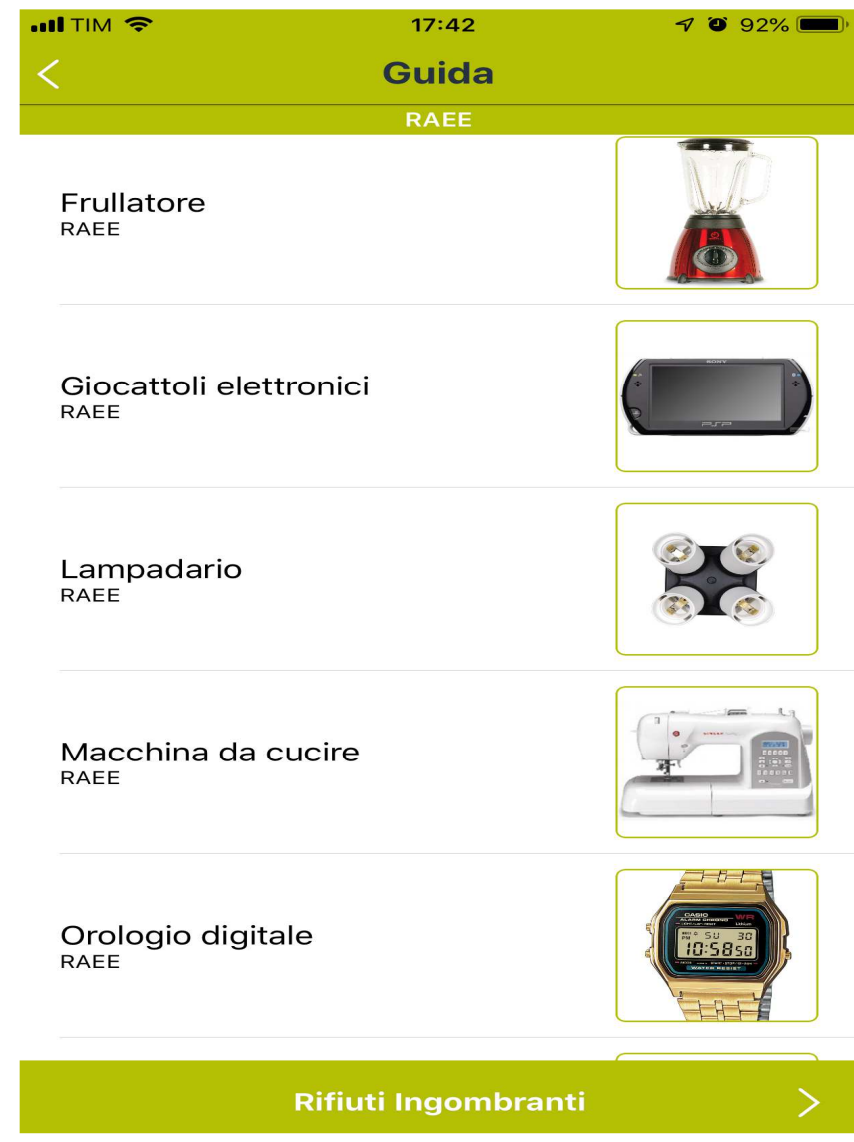

Fig. 9: greenBag App Dictionary

The first test of the green Bag solution has been performed in the South of Italy, thanks to a local UCO collection company. It usually collects ca. $670 \mathrm{~kg}$ of UCO per month, implementing a door-to-door methodology. During the two months of greenBag test campaign, performed in June and July, it advised people about the suspension of the door-to-door service and about the availability of a Bin inside the city. The same day, NFC cards were provided to people and a workshop, explaining how to use the Bin, was arranged.

During June, the rate of UCO collection increased of $80 \mathrm{~kg}$ and during July, this rate improved to $150 \mathrm{~kg}$. The test bed has been dismissed after July. The implementation of greenBag brought an increase of ca. the $17 \%$ of the UCO collected considering both June and July; but considering only July, one month after the greenBag installation (when people were now confident with the system), the increase was of ca. $23 \%$.

\section{CONCLUSION}

The greenBag solution is a complex platform able to identify the waste disposed inside of it and the people who provide this waste. It can be used to improve people efficiency in waste separation but it can be also used to optimize the effort of the waste collection company.

\section{REFERENCES}

[1] Faizal, Muhammad. "Utilization Biomass and Coal Mixture to Produce Alternative Solid Fuel for Reducing Emission of Green
House Gas." International Journal on Advanced Science, Engineering, and Information Technology 7.3 (2017): 950-956.

[2] Anggraini, Tuty, Sahadi Didi Ismanto, and Dahlia Dahlia. "The Making of Transparent Soap from Green Tea Extract." International Journal on Advanced Science, Engineering, and Information Technology 5.4 (2015): 349-356.

[3] Fatriasari, Widya, et al. "Lignin and cellulose changes of betung bamboo (Dendrocalamus asper) pretreated microwave heating." International Journal on Advanced Science, Engineering, and Information Technology 6.2 (2016): 186-195.

[4] Capizzi, Giacomo, et al. "Cascade feed forward neural network-based model for air pollutants evaluation of single monitoring stations in urban areas." International Journal of Electronics and Telecommunications 61.4 (2015): 327-332.

[5] Greenea, Analysis of the current development of household UCO collection systems in the EU 23 May 2016

[6] Tsay M, "The Impact of Double Counting Legislation on UCOME \& TME. 1st Annual Biofuels Conference 2012-Tackling Fragmentation in the International Biofuels Sector". June 28-29, 2012, Amsterdam

[7] Matta, M., Cardarilli, G.C., Di Nunzio, L., Fazzolari, R., Giardino, D., Re, M., Silvestri, F., Spanò, S. Q-RTS: A real-time swarm intelligence based on multi-agent Q-learning (2019) Electronics Letters, 55 (10), pp. 589-591.

[8] Gian Carlo Cardarilli, Luca Di Nunzio, Rocco Fazzolari, Daniele Giardino, Marco Matta, Marco Re, Francesca Silvestri and Sergio Spanò Efficient Ensemble Machine Learning implementation on FPGA using Partial Reconfiguration (2019) Lecture Notes in Electrical Engineering ARTICLE IN PRESS

[9] Ammendola, Roberto, and Pierpaolo Loreti. "Design and Evaluation of a Scalable Engine for 3D-FFT Computation in an FPGA Cluster." International Journal on Advanced Science, Engineering, and Information Technology 9.2 (2019): 677-684.

[10] Gian Carlo Cardarilli, Luca Di Nunzio, Rocco Fazzolari, Marco Re, Sergio Spanó AW-SOM, an Algorithm for High-speed Learning in Hardware Self-Organizing Maps IEEE Transactions on Circuits and Systems II

[11] Susi, Gianluca, et al. "A Neuro-Inspired System for Online Learning and Recognition of Parallel Spike Trains, Based on Spike Latency, and Heterosynaptic STDP." Frontiers in neuroscience 12 (2018).

[12] Gian Carlo Cardarilli; Luca Di Nunzio; Rocco Fazzolari; Alberto Nannarelli; Marco Re; Sergio Spanò N-Dimensional Approximation of Euclidean Distance IEEE Transactions on Circuits and Systems II: Express Briefs ARTICLE IN PRESS

[13] Detti, Andrea, et al. "Application of information centric networking to nosql databases: the spatio-temporal use case." 2017 IEEE International Symposium on Local and Metropolitan Area Networks (LANMAN). IEEE, 2017.

[14] Bracciale, Lorenzo, et al. "Lightweight Named Object: an ICN-based Abstraction for IoT Device Programming and Management." IEEE Internet of Things Journal (2019).

[15] Loreti, P., Catini, A., De Luca, M., Bracciale, L., Gentile, G. and Di Natale, C., 2019. "The Design of an Energy Harvesting Wireless Sensor Node for Tracking Pink Iguanas". Sensors, 19(5), p.985.

[16] Bracciale, L., Catini, A., Gentile, G., Loreti, P. Delay tolerant wireless sensor network for animal monitoring: The Pink Iguana case (2017) Lecture Notes in Electrical Engineering, 429, pp. 18-26.

[17] Beritelli, Francesco, et al. "Automatic heart activity diagnosis based on Gram polynomials and probabilistic neural networks." Biomedical engineering letters 8.1 (2018): 77-85.

[18] Silvestri, F., Acciarito, S., Cardarilli, G.C., Khanal, G.M., Di Nunzio, L., Fazzolari, R., Re, M. FPGA implementation of a low-power QRS extractor (2019) Lecture Notes in Electrical Engineering, 512, pp. 915 .

[19] Makky, Muhammad, and Peeyush Soni. "Towards sustainable green production: exploring automated grading for oil palm fresh fruit bunches (FFB) using machine vision and spectral analysis." International Journal on Advanced Science, Engineering and Information Technology 3.1 (2013): 1-5.

[20] Capizzi, G., and G. Tina. "Long-term operation optimization of integrated generation systems by fuzzy logic-based management." Energy 32.7 (2007): 1047-1054 